
\title{
28 Research Suare \\ Upregulation of RAB7 Relates To Neuronal Pyroptosis After Spinal Cord Injury In Rat
}

Chao Liu

The Second Affiliated Hospital of Soochow University

Qianliang Wang

The Second Affiliated Hospital of Soochow University

\section{Sunao Li}

Soochow University

\section{Xueshi Chen}

Soochow University

\section{Xinqi Huang}

Soochow University

Jun Yan ( $\square$ liuchaotougao@163.com )

The Second Affiliated Hospital of Soochow University https://orcid.org/0000-0003-1970-1600 Haiyan Shan

The Affiliated Suzhou Hospital of Nanjing Medical University

\section{Mingyang Zhang}

Soochow University

\section{Research Article}

Keywords: Rab7, Pyroptosis, Spinal Cord Injury.

Posted Date: January 28th, 2022

DOI: https://doi.org/10.21203/rs.3.rs-1219162/v1

License: (c) (i) This work is licensed under a Creative Commons Attribution 4.0 International License. Read Full License 


\section{Abstract}

The Rab7 belongs to the Ras small GTPase superfamily, and the abnormal expression of Rab7 can cause neuropathy and lipid metabolism disease. However, the regulatory mechanism was not explicitly clear. Studies show that its plays crucial role inner membrane translocase. However, the role of Rab7 on the regulatory mechanisms of cell survival is still unknown. We performed a rat spinal cord injury (SCI) model to explore the cellular localization and expression of Rab7 after SCl in this study. Western blot has found that Rab7 was expressed in the spinal cord tissue. On the first day, it significantly increased, then decreased at $48 \mathrm{~h}$, and on the third day reached a peak after SCl. what important is that Rab7 was colocalized with neurons. Furthermore, Western blot was also demonstrated that the pyroptosis-related protein (GSDMD , Caspase-1, ASC) expression attained the peak after the third-day post-injury.

Importantly, the immunohistochemistry analysis revealed that Rab7 was completely colocalized with ASC in the neuron after $\mathrm{SCl}$. These results suggested that Rab7 was colocalized with the neurons and involved in the pyroptosis of neurons and closely related to the spinal cord after injury.

\section{Introduction}

$\mathrm{SCl}$ is a devastating disease, an affliction of the nervous system, results in disturbed sensory, autonomic, and motor functions and a financial burden (Anjum et al. 2020). SCl may be divided into both primary and secondary mechanisms of injury. During $\mathrm{SCl}$, most of the cells died because of injury-induced biochemical changes (secondary injury). The secondary injury induced by SCl leads to cell death may persist for several days to weeks and cause nervous malfunction (Zhu et al. 2017). Studies have demonstrated that apoptosis, inflammation, tissue ischemia, cell edema, and other pathophysiological converts can greatly reinforce the damage level of SCI which leads to multiple neurological dysfunctions after trauma (Alizadeh et al. 2019; Alizadeh et al. 2017). Neuroinflammation largely causes a series of secondary injury which finally lead to the neuronal death process (Ringel-Scaia et al. 2016; Simon et al. 2017). Pyroptosis is an inflammation-dependent programmed cell death and is affected by the activation of GSDMD, caspase-4/5/11 and caspase-1, and produces various inflammatory mediators such as IL-18 (He et al. 2015; Jorgensen and Miao 2015; Ruan 2019). It has previously been demonstrated to serve a crucial role in the pathogenesis of SCI (Al Mamun et al. 2021). However, the mechanism of pyroptosis in $\mathrm{SCl}$ is still poorly understood.

The Rab proteins are one of the Ras small GTPase superfamilies, a large amount of literature has shown that Rab proteins are key regulators of membrane trafficking, cell signaling, cell growth and development by binding to effecter proteins (Gonzalez-Gaitan and Stenmark 2003; Snider 2003). Rab7 has been regarded as an important factor in endosomal membrane trafficking in neuronal cells (Bucci et al. 2000). Additionally, Rab7 was found localized in the lysosome, and the late endosomal membrane fusion and trafficking were regulated by Rab7 (Chavrier et al. 1990; Zhang et al. 2009).

Studies demonstrated that Rab proteins are related to neurological functions and expressed in glia and neurons, and the mutation or disfunction of Rab7 may cause neurological disorders ( $\mathrm{Ng}$ and Tang 2008). 
Cataldo's reports have shown that the defects of Rab7, which regulates late endocytic traffic, are associated with Down's syndrome and Alzheimer's disease (Cataldo et al. 2008). In neurons, Axonal transport is key for its survival and differentiation and helps to long-range communication of neurons. Saxena, et al (Saxena et al. 2005)showed that, in the endosome, inhibiting Rab7 activity leads to aggregation of tropomyosin receptor kinase A (TrkA) and potentiated NGF-stimulated signaling of TrkA to advance neurite outgrowth. These data indicate that Rab7 has a crucial position in the central nervous system (CNS). However, the role of Rab7 in spinal cord function has not been investigated. Furthermore, currently, most studies are performed in vitro with cells. The experimental with animal models may have additional insight on the underlying mechanisms of Rab7 in neuropathy.

In our study, we show the distribution and expression of Rab7 in the spinal of rats after $\mathrm{SCl}$, and first investigate the relation between Rab7 and pyroptosis after SCl. Aimed to gain a deep perception about the Rab7 functions in injured and normal spinal and its roles in the molecular and cellular mechanisms underlying nervous system damage and repair.

\section{Materials And Methods}

\section{Animals and $\mathrm{SCl}$ model}

Male adult Sprague Dawley (SD) rats weighing 220-250g (6-8 weeks old) were used in this study, and those rats were gained from the Laboratory Animal Center of Soochow University. They are placed with constant temperature and humidity, and a 12-hour light-dark cycle. All procedures about animals were permitted by the Institutional Animal Use and Care Committee at Soochow University and the China Laboratory Animal Guidelines. In experiments, data were obtained by investigators blinded to the study group. In addition, all experiments were conducted during the daytime (7:00-19:00). The most important point is that all efforts were taken to obtain minimal suffering and reduction for animal use.

In the $\mathrm{SCl}$ group, the rats were established by using the modified weight-drop method as previously described (Dai et al. 2019). All animals were divided into two experimental groups, randomly, with 6 rats per group: Sham group, SCl group ( $1 \mathrm{~h}, 6 \mathrm{~h}, 12 \mathrm{~h}, 1 \mathrm{~d}, 2 \mathrm{~d}, 3 \mathrm{~d}, 7 \mathrm{~d})$. Rats were anesthetized by injecting pentobarbital sodium $(50 \mathrm{mg} / \mathrm{kg}$ ) into the peritoneum. Briefly, The T8-T10 lamina was removed after exposure, and the spinal cord was exposed, fully. Laminectomy was operated at the T9 level. We use a dropping bar which was $9 \mathrm{~g}$ and $2.5 \mathrm{~mm}$ in diameter to hit the exposed spinal cord from a height of 6.0 $\mathrm{cm}$, The spinal cord hemorrhage, the delayed extension of hind limbs and tail swing indicated the successful establishment of the SCI model. Subsequently, the incision was sutured layer by layer. Signs

of successful modeling included spinal cord hemorrhage, convulsions in hind limbs and tail swing. Until spontaneous urination was resolved, the bladder of rats was evacuated manually. The spinal cord specimens were obtained for the follow-up experiments.

\section{Western Blot Analysis}


An overdose of chloralhydrate was used to treat rats and then be killed at different points $(n=6)$; the spinal cords $(1 \mathrm{~cm})$ were removed and stored at $-80^{\circ} \mathrm{C}$ until further use. Proteins were distilled from spinal cord tissue in RIPA lysis buffer. Then use an enhanced BCA protein assay kit to determine the concentrations of protein. Equal quantities of protein were divided via sodium dodecyl sulfatepolyacrylamide gel electrophoresis (SDS-PAGE), and then transferred onto polyvinylidene difluoride (PVDF) membranes. and the PVDF was then blocked with 5\% BSA for $2 \mathrm{~h}$ at room temperature, and then the PVDF was incubated with the relative primary antibodies overnight at $4^{\circ} \mathrm{C}$. The primary antibodies were as follows: Anti-Rab7(1:400), Anti-GSDMD (1:500), Anti-Caspase 1 (1:100), and Anti-ASC (1:200) antibodies were acquired from Santa Cruz Biotechnology. After washing by TBST, blots were incubated with secondary antibodies at normal temperature for $2 \mathrm{~h}$. the ECL chemiluminescence system was used to measure the blots and then captured on autoradiographic films. The films were then scanned. The densitometric analysis of the bands was conducted with Image $\mathrm{J}$ software.

\section{Sections and Immunohistochemistry}

The chloral hydrate ( $10 \%$ solution) was used to anesthetize rats, terminally. Then the rats were perfused with $50 \mathrm{~mL}$ of $0.01 \mathrm{M}$ PBS, pericardially, and finally used $4 \%$ paraformaldehyde. The spinal cord was removed after perfusion and postfixed in the fixative for $12 \mathrm{~h}$, and then $20 \%$ and $30 \%$ sucrose for 1 day, respectively. An optimal cutting temperature compound was used to embed the spinal cords. $5-\mu \mathrm{m}$ frozen cross-sections were prepared for the examination. The slices can be frozen in a refrigerator at $-80^{\circ} \mathrm{C}$. The sections were blocked with bovine serum albumin (BSA) for $2 \mathrm{~h}$ at normal temperature. The anti-Rab7 antibody (anti-mouse, 1:100) was used to incubate the sections overnight at $4{ }^{\circ} \mathrm{C}$, then the sections were incubated in a related secondary antibody (Vector Laboratories, Burlingame, CA, USA). The DAB (Vector Laboratories) was used to visualize staining, The strong or moderate brown staining of cells was regarded as positive, cells with weak staining were scored separately, while cells were regarded as negative when with no staining.

\section{Double Immunofluorescent Staining}

To avoid the sections unspecific staining, $10 \%$ normal serum blocking solution which contains BSA and Triton X-100, and Tween-20 was used to block at room temperature for $2 \mathrm{~h}$, and the sections were then incubated with primary antibodies for anti-ASC (1:50; Abcam; rabbit), antibodies for anti-Rab7 (1:50; Santa Cruz; mouse), anti-NeuN(1:500; Sigma), anti-GFAP(1:500; Sigma), anti-IBA1(1:500; Sigma). Above all, the primary antibodies were used to incubate the sections overnight at $4{ }^{\circ} \mathrm{C}$, then a mixture of fluorescein isothiocyanate and tetramethylrhodamine of secondary antibodies were used to incubate at room temperature for $2 \mathrm{~h}$. Leica Spectral Confocal microscope (Germany) was used to examine the stained sections.

\section{Statistical Analysis}

Statistical analyses were dealt with GraphPad8.0. Results are presented as means \pm SEM. The statistical was determined with one-way analysis of variance (ANOVA) followed by Student t-test (two means 
comparison) or Tukey's post hoc multiple comparison tests. $\mathrm{P}<0.05$ was considered significant.

\section{Result}

\section{The Rab7 Expression Following SCI}

To identify the Rab7 expression pattern, the expression of Rab7 protein was examined by Western blot at different time points after SCl and in sham control. In the spinal cord around the lesion site, Rab7 protein markedly increased on the $24 \mathrm{~h}$ compared to sham groups, then decreased at $48 \mathrm{~h}$, and attained a peak on day 3 (Fig. 1).

\section{The Rab7 Immunoreactivity Staining In Rat After Sci}

To recognize the Rab7 immunoreactivity changes in medulla spinalis three days after spinal cord injury, an anti-Rab7 mouse monoclonal antibody was used to perform the immunohistochemistry for transverse cryosections of myeloid tissue, $5 \mathrm{~mm}$ around to the damaged site. Markedly, Rab7 staining was expressed in the myeloid tissue (Fig. 2). In addition, Rab7 expression was estimated by counting cells between sham and spinal cord injury groups (Fig. 2); the results were comparable with Western blot (Fig. 1).

\section{Expression of the Neuronal Pyroptosis In Spinal Cord After Injured}

To confirm the links between Rab7 and pyroptosis cells, the studies were performed as follows: The level of pyroptosis-related proteins (ASC, Caspase-1, GSDMD) in myeloid tissue was detected by immunoblotting. Those proteins activation occurred in the myeloid tissue of rats after $\mathrm{SCl}$ with outstanding activation occurring $24 \mathrm{~h}$ post-injury, and reaching the peak at day 3 after SCl (Fig. 3). To reveal the pyroptosis cell types after $\mathrm{SCl}$, the double immunofluorescence staining was with various phenotype-specific markers and ASC-specific antibodies (Fig. 5). On the third day, most of the reactive neurons were ASC-positive (Fig. 5). The Rab7-expressing neurons were partly revealed as ASC positive (Fig. 4). And, double immunofluorescent staining indicated that Rab7 was colocalized with ASC (Fig. 6).

\section{Discussion}

To reveal the distribution and expression of Rab7 in the medulla spinalis of rats after injury, we established the animals' SCI models. In our experiment, we elucidated the upregulation of Rab7 in rat medulla spinalis after SCl. Rab7 was obviously observed in the rat spinal cord and specifically expressed in the neurons of the spinal cord, however, the detectable levels of Rab7 were not be observed in astrocytes and microglia. The immunohistochemistry demonstrated that Rab7 is strongly expressed in the $\mathrm{SCl}$ group compared with the sham group. Furthermore, immunoblot analysis demonstrated that the protein level of Rab7 gets to a peak on day 3 after SCl. Surprisingly, we also observed that pyroptosis cells marker ASC specifically expressed in the neurons of the spinal cord and there was also peak 
accumulation of ASC at 3 days post-injury. To confirm the links between Rab7 and pyroptosis cells, we performed double immunofluorescence staining with Rab7 and ASC. The result revealed that Rab7expressing neurons were partly shown as ASC positive. In addition, fluorescence microscopic immunolabeling of ASC in spinal sections demonstrated that neuronal undergo pyroptosis after $\mathrm{SCl}$. To sum up, it showed that in the traumatic spinal cord, the expression of Rab7 may be induced in the preoptic neurons after SCl. These data may provide significant insights to understanding the cellular and molecular mechanisms underlying SCl.

It was reported that Rab GTPases was the largest family of small GTP-binding proteins that contains more than 60 members in the human genome (Yang et al. 2015). Rab7 is one of this large family and has a unique role in modulating autophagy (Wen et al. 2017). Autophagy is a catabolic process via degrading protein aggregates, cytoplasmic proteins and organelles, and is tightly correlated to cellular metabolism (Mizushima et al. 2008; Sun et al. 2018). After SCl, the markers of autophagy were increased, however, their mechanisms and functions remain controversial. Excessive autophagic activation evokes autophagic programmed cell death (Pi et al. 2019). Although autophagy was increased under certain conditions pathologically and has been implicated in cell death(Luo and Tao 2020), under most conditions autophagy is considered to be cytoprotective. Many studies have shown that increasing autophagy can protect neurons by down-regulating the level of apoptosis (Chen et al. 2018; Zhang et al. 2019; Zhao et al. 2017). Gao $C$ et al also report that autophagy activation could have a neuroprotective effect by repressing pyroptosis in a Mouse Model of Moderate Traumatic Brain Injury(Gao et al. 2020). Thus, Rab7 May play a role in pyroptosis through regulating autophagy and needs further study.

Pyroptosis, the other form of programmed necrosis, is a pro-inflammatory form of regulated cell death that depends on the enzymatic activity of inflammatory proteases that pertain to the cysteine-dependent aspartate-specific protease (caspase) family. Pyroptosis is mainly observed in professional phagocytes of the myeloid lineage, The higher levels of expression of inflammatory caspases of these cell types may be the possible mechanism. However, it has also been observed in neurons and other cells. The occurrence of pyroptosis depends on the activation of the inflammasome which leads to the recruitment of the adaptor protein ASC. In many studies, ASC can be one of the reliable markers for pyroptosis. Previous studies indicated that pyroptosis markers increased in the spinal after injury (Li et al. 2020; Liu et al. 2020; Wu et al. 2020). In our experiment, the effect of SCl on regional cellular patterns of expression of ASC was detected. These results are consistent with ASC activation that appeared in the spinal cord after SCl. However, the timing sequence of pyroptosis in $\mathrm{SCl}$ has not previously been reported incomparable detail. In our study, on the first day, the pyroptosis-related proteins significantly increased and then attained a peak on day 3 after SCl. Interestingly, those proteins then decreased at $48 \mathrm{~h}$, This might be related to the mechanisms mediating the autoimmune response after $\mathrm{SCl}$. Studies demonstrated that the vascular immediate disrupt after injury and then trigger secondary pathology, the immune cells extravasated around the lesion site conduct pressure on the damaged spinal tissues and destroy the blood flow, fourthly, this state continues up to $24 \mathrm{~h}$ (Alizadeh et al. 2019). Due to different injuries in the spinal cord injury animal model, the timing sequence of pyroptosis in $\mathrm{SCl}$ remains inconclusive. Therefore, it will be of great value to conduct an in-depth exploration of it. 
Evidence showed that pyroptosis played an important function in the pathogenesis of multiple neurological disorders as well as SCI (Al Mamun et al. 2021). Compared with the changes of pyroptosisrelated proteins after $\mathrm{SCl}$, Rab7 was also region-specific and time-dependent. the neurons of Rab7 expressing partly showed as well as ASC positive. Thus, we hypothesized that upregulation of Rab7 in the spinal cord was strong links to the pyroptosis-related proteins after $\mathrm{SCl}$, suggesting a role that Rab7 may play a critical function in pyroptosis after $\mathrm{SCl}$

Above all, the evidence indicated that Rab7 has an important function in the process of pyroptosis induced by SCl. We first showed the expression profile of Rab7 in rat spinal cord after SCl. Our results may provide significant insights to understand the underlying cellular and molecular mechanisms of CNS after $\mathrm{SCl}$ and a new strategy for the treatment of $\mathrm{SCl}$. The function of Rab7 on cellular pyroptosis, however, still needs further research, and future studies also attempt to explore their possible substrates and downstream signaling targets.

\section{Declarations}

\section{Conflicts of Interest}

The authors declare no conflict of interest.

\section{Authors' Contributions}

Chao Liu and Qianliang Wang contributed equally to this work.

\section{Acknowledgments}

This work was supported by the National Natural Science Foundation of China (No. 82071382, No.81601306); The Priority Academic Program Development of Jiangsu Higher Education Institutions (PAPD); The Jiangsu Maternal and Child Health Research Key Project (F202013); Jiangsu Talent Youth Medical Program (QNRC2016245); Shanghai Key Lab of Forensic Medicine (KF2102); Suzhou Science and Technology Development Project (SYS2020089, SYS2018082); The Fifth Batch of Gusu District Health Talent Training Project (GSWS2019060); the National Natural Science Foundation of China (grant nos. 81902239 and 81971036) and the Natural Science Foundation of Jiangsu Province (grant no. BK20191169); The Science and Technology Development Fund of Nanjing Medical University \NMUB2019248邓; Hospital project of Suzhou BenQ Medical Center(SZMJ18005).

\section{Data Availability}

All data generated or analysed during this study are included in this published article (and its supplementary information files).

Ethics approval and consent to participate: Our study was approved by the Ethical Committee of Soochow University. 
Consent for publication: All of the authors have given their consent for publication

Availability of data and materials: All data generated or analysed during this study are included in this published article (and its supplementary information files).

Competing interests: The authors declare that they have no competing interests.

Funding: This work was supported by the National Natural Science Foundation of China (No. 82071382, No.81601306); The Priority Academic Program Development of Jiangsu Higher Education Institutions (PAPD); The Jiangsu Maternal and Child Health Research Key Project (F202013); Jiangsu Talent Youth Medical Program (QNRC2016245); Shanghai Key Lab of Forensic Medicine (KF2102); Suzhou Science and Technology Development Project (SYS2020089, SYS2018082); The Fifth Batch of Gusu District Health Talent Training Project (GSWS2019060); the National Natural Science Foundation of China (grant nos. 81902239 and 81971036) and the Natural Science Foundation of Jiangsu Province (grant no. BK20191169); The Science and Technology Development Fund of Nanjing Medical University \NMUB2019248囚; Hospital project of Suzhou BenQ Medical Center(SZMJ18005).

Authors' contributions: Chao Liu, Qianliang Wang wrote and designed the structure of the manuscript. Sunao Li, Xueshi Chen and Xinqi Huang managed the literature searches and analyses. Jun Yan, Haiyan Shan and Mingyang Zhang assisted with the improvement of the manuscript

Acknowledgments: The authors thank Jun Yan, Haiyan Shan, and Mingyang Zhang for assistance with final corrections, and Sunao Li, Xueshi Chen and Xinqi Huang for help in the literature search.

Authors' information (optional): Chao Liu ${ }^{1,4}$, Qianliang Wang ${ }^{1}$, Sunao $\mathrm{Li}^{3}$, Xueshi Chen ${ }^{3}$, Xinqi Huang $^{3}$, Jun Yan ${ }^{1}$, Haiyan Shan ${ }^{2}$, Mingyang Zhang ${ }^{3}$,

${ }^{1}$ Department of Orthopedics, The Second Affiliated Hospital of Soochow University, Suzhou, China

2 Department of Obstetrics and Gynecology, The Affiliated Suzhou Hospital of Nanjing Medical University, Suzhou, China;

${ }^{3}$ Department of Forensic Sciences, School of Basic Medicine and Biological Sciences, Soochow University, Suzhou, China;

${ }^{4}$ Department of Orthopedics, Suzhou BenQ Medical Center, The Affiliated BenQ Hospital of Nanjing Medical University, Suzhou, China;

*Correspondence to Dr. Jun Yan, Department of Orthopedics, The Second Affiliated Hospital of Soochow University, Suzhou, 215123 Jiangsu, China E-mail: cxyanjun@hotmail.com

Dr. Haiyan Shan, Department of Obstetrics and Gynecology, The Affiliated Suzhou Hospital of Nanjing Medical University, Suzhou, China E-mail: ghostqth@163.com 
Dr. Mingyang Zhang, Department of Forensic Sciences, Soochow University, Suzhou 215123 Jiangsu, China E-mail: mingyangzhang@suda.edu.cn

\section{References}

1. Al Mamun A, Wu Y, Monalisa I, Jia C, Zhou K, Munir F, Xiao J (2021) Role of pyroptosis in spinal cord injury and its therapeutic implications. J Adv Res 28:97-109. https://dx.doi.org/10.1016/j.jare.2020.08.004

2. Alizadeh A, Dyck SM, Karimi-Abdolrezaee S (2019) Traumatic Spinal Cord Injury: An Overview of Pathophysiology, Models and Acute Injury Mechanisms. Front Neurol 10:282. https://dx.doi.org/10.3389/fneur.2019.00282

3. Alizadeh A, Dyck SM, Kataria H, Shahriary GM, Nguyen DH, Santhosh KT, Karimi-Abdolrezaee S (2017) Neuregulin-1 positively modulates glial response and improves neurological recovery following traumatic spinal cord injury. Glia 65:1152-1175. https://dx.doi.org/10.1002/glia.23150

4. Anjum A, Yazid MD, Fauzi Daud M, Idris J, Ng AMH, Selvi Naicker A, Ismail OHR, Athi Kumar RK, Lokanathan Y (2020) Spinal Cord Injury: Pathophysiology, Multimolecular Interactions, and Underlying Recovery Mechanisms. Int J Mol Sci 21. https://dx.doi.org/10.3390/ijms21207533

5. Bucci C, Thomsen P, Nicoziani P, McCarthy J, van Deurs B (2000) Rab7: a key to lysosome biogenesis. Mol Biol Cell 11:467-480. https://dx.doi.org/10.1091/mbc.11.2.467

6. Cataldo AM, Mathews PM, Boiteau AB, Hassinger LC, Peterhoff CM, Jiang Y, Mullaney K, Neve RL, Gruenberg J, Nixon RA (2008) Down syndrome fibroblast model of Alzheimer-related endosome pathology: accelerated endocytosis promotes late endocytic defects. Am J Pathol 173:370-384. https://dx.doi.org/10.2353/ajpath.2008.071053

7. Chavrier P, Parton RG, Hauri HP, Simons K, Zerial M (1990) Localization of low molecular weight GTP binding proteins to exocytic and endocytic compartments. Cell 62:317-329. https://dx.doi.org/10.1016/0092-8674(90)90369-p

8. Chen X, Pan Z, Fang Z, Lin W, Wu S, Yang F, Li Y, Fu H, Gao H, Li S (2018) Omega-3 polyunsaturated fatty acid attenuates traumatic brain injury-induced neuronal apoptosis by inducing autophagy through the upregulation of SIRT1-mediated deacetylation of Beclin-1. J Neuroinflammation 15:310. https://dx.doi.org/10.1186/s12974-018-1345-8

9. Dai W, Wang X, Teng H, Li C, Wang B, Wang J (2019) Celastrol inhibits microglial pyroptosis and attenuates inflammatory reaction in acute spinal cord injury rats. Int Immunopharmacol 66:215223. https://dx.doi.org/10.1016/j.intimp.2018.11.029

10. Gao C, Yan Y, Chen G, Wang T, Luo C, Zhang M, Chen X, Tao L (2020) Autophagy Activation Represses Pyroptosis through the IL-13 and JAK1/STAT1 Pathways in a Mouse Model of Moderate Traumatic Brain Injury. ACS Chem Neurosci 11:4231-4239. https://dx.doi.org/10.1021/acschemneuro.0c00517 
11. Gonzalez-Gaitan M, Stenmark H (2003) Endocytosis and signaling: a relationship under development. Cell 115:513-521. https://dx.doi.org/10.1016/s0092-8674(03)00932-2

12. He WT, Wan H, Hu L, Chen P, Wang X, Huang Z, Yang ZH, Zhong CQ, Han J (2015) Gasdermin D is an executor of pyroptosis and required for interleukin-1beta secretion. Cell Res 25:1285-1298. https://dx.doi.org/10.1038/cr.2015.139

13. Jorgensen I, Miao EA (2015) Pyroptotic cell death defends against intracellular pathogens. Immunol Rev 265:130-142. https://dx.doi.org/10.1111/imr.12287

14. Li X, Yu Z, Zong W, Chen P, Li J, Wang M, Ding F, Xie M, Wang W, Luo X (2020) Deficiency of the microglial Hv1 proton channel attenuates neuronal pyroptosis and inhibits inflammatory reaction after spinal cord injury. J Neuroinflammation 17:263. https://dx.doi.org/10.1186/s12974-020-01942$\mathrm{x}$

15. Liu Z, Yao X, Jiang W, Li W, Zhu S, Liao C, Zou L, Ding R, Chen J (2020) Advanced oxidation protein products induce microglia-mediated neuroinflammation via MAPKs-NF-kappaB signaling pathway and pyroptosis after secondary spinal cord injury. J Neuroinflammation 17:90. https://dx.doi.org/10.1186/s12974-020-01751-2

16. Luo C, Tao L (2020) The Function and Mechanisms of Autophagy in Spinal Cord Injury. Adv Exp Med Biol 1207:649-654. https://dx.doi.org/10.1007/978-981-15-4272-5_47

17. Mizushima N, Levine B, Cuervo AM, Klionsky DJ (2008) Autophagy fights disease through cellular self-digestion. Nature 451:1069-1075. https://dx.doi.org/10.1038/nature06639

18. Ng EL, Tang BL (2008) Rab GTPases and their roles in brain neurons and glia. Brain Res Rev 58:236-246. https://dx.doi.org/10.1016/j.brainresrev.2008.04.006

19. Pi H, Li M, Zou L, Yang M, Deng P, Fan T, Liu M, Tian L, Tu M, Xie J, Chen M, Li H, Xi Y, Zhang L, He M, Lu Y, Chen C, Zhang T, Wang Z, Yu Z, Gao F, Zhou Z (2019) AKT inhibition-mediated dephosphorylation of TFE3 promotes overactive autophagy independent of MTORC1 in cadmiumexposed bone mesenchymal stem cells. Autophagy 15:565-582. https://dx.doi.org/10.1080/15548627.2018.1531198

20. Ringel-Scaia VM, McDaniel DK, Allen IC (2016) The Goldilocks Conundrum: NLR Inflammasome Modulation of Gastrointestinal Inflammation during Inflammatory Bowel Disease. Crit Rev Immunol 36:283-314. https://dx.doi.org/10.1615/CritRevImmunol.2017019158

21. Ruan J (2019) Structural Insight of Gasdermin Family Driving Pyroptotic Cell Death. Adv Exp Med Biol 1172:189-205. https://dx.doi.org/10.1007/978-981-13-9367-9_9

22. Saxena S, Bucci C, Weis J, Kruttgen A (2005) The small GTPase Rab7 controls the endosomal trafficking and neuritogenic signaling of the nerve growth factor receptor TrkA. J Neurosci 25:1093010940. https://dx.doi.org/10.1523/JNEUROSCI.2029-05.2005

23. Simon DW, McGeachy MJ, Bayir H, Clark RSB, Loane DJ, Kochanek PM (2017) The far-reaching scope of neuroinflammation after traumatic brain injury. Nat Rev Neurol 13:572. https://dx.doi.org/10.1038/nrneurol.2017.116 
24. Snider MD (2003) A role for rab7 GTPase in growth factor-regulated cell nutrition and apoptosis. Mol Cell 12:796-797. https://dx.doi.org/10.1016/s1097-2765(03)00401-5

25. Sun Y, Xia M, Yan H, Han Y, Zhang F, Hu Z, Cui A, Ma F, Liu Z, Gong Q, Chen X, Gao J, Bian H, Tan Y, Li $Y$, Gao X (2018) Berberine attenuates hepatic steatosis and enhances energy expenditure in mice by inducing autophagy and fibroblast growth factor 21. Br J Pharmacol 175:374-387. https://dx.doi.org/10.1111/bph.14079

26. Wen H, Zhan L, Chen S, Long L, Xu E (2017) Rab7 may be a novel therapeutic target for neurologic diseases as a key regulator in autophagy. $J$ Neurosci Res 95:1993-2004. https://dx.doi.org/10.1002/jnr.24034

27. Wu C, Xu H, Li J, Hu X, Wang X, Huang Y, Li Y, Sheng S, Wang Y, Xu H, Ni W, Zhou K (2020) Baicalein Attenuates Pyroptosis and Endoplasmic Reticulum Stress Following Spinal Cord IschemiaReperfusion Injury via Autophagy Enhancement. Front Pharmacol 11:1076. https://dx.doi.org/10.3389/fphar.2020.01076

28. Yang Y, Hou N, Wang X, Wang L, Chang S, He K, Zhao Z, Zhao X, Song T, Huang C (2015) miR-15b-5p induces endoplasmic reticulum stress and apoptosis in human hepatocellular carcinoma, both in vitro and in vivo, by suppressing Rab1A. Oncotarget 6:16227-16238. https://dx.doi.org/10.18632/oncotarget.3970

29. Zhang M, Chen L, Wang S, Wang T (2009) Rab7: roles in membrane trafficking and disease. Biosci Rep 29:193-209. https://dx.doi.org/10.1042/BSR20090032

30. Zhang Y, Zhang Y, Jin XF, Zhou XH, Dong XH, Yu WT, Gao WJ (2019) The Role of Astragaloside IV against Cerebral Ischemia/Reperfusion Injury: Suppression of Apoptosis via Promotion of P62-LC3Autophagy. Molecules. 24https://dx.doi.org/10.3390/molecules24091838

31. Zhao H, Chen S, Gao K, Zhou Z, Wang C, Shen Z, Guo Y, Li Z, Wan Z, Liu C, Mei X (2017) Resveratrol protects against spinal cord injury by activating autophagy and inhibiting apoptosis mediated by the SIRT1/AMPK signaling pathway. Neuroscience 348:241-251. https://dx.doi.org/10.1016/j.neuroscience.2017.02.027

32. Zhu Z, Shen Q, Zhu L, Wei X (2017) Identification of pivotal genes and pathways for spinal cord injury via bioinformatics analysis. Mol Med Rep 16:3929-3937. https://dx.doi.org/10.3892/mmr.2017.7060

\section{Figures}



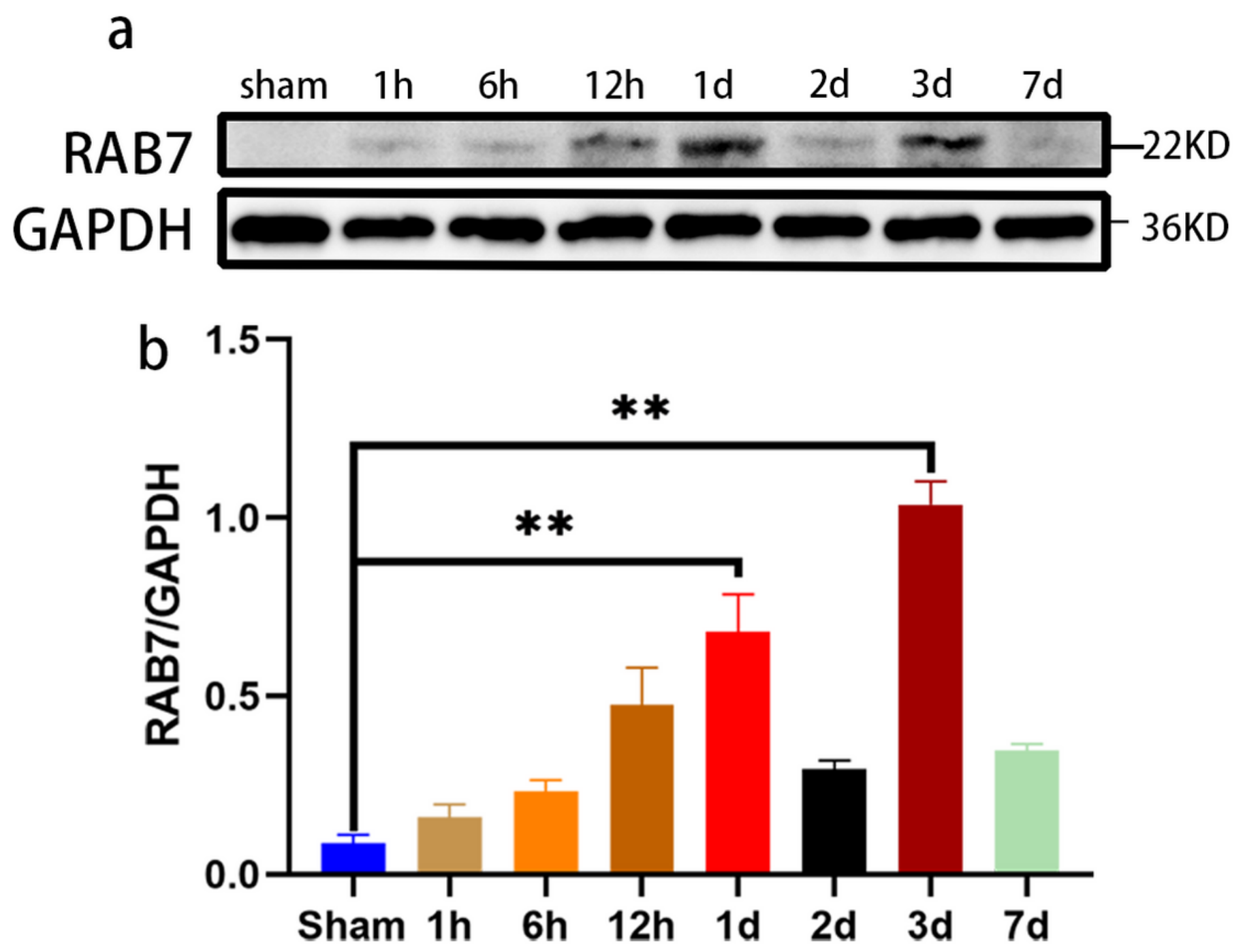

Figure 1

The Rab7 expression changes after SCl.

(a)spinal cords of rats were extracted at different times after $\mathrm{SCl}$ for analysis. Immunoblots probed for Rab7 and GAPDH are shown. The bar chart below indicates the ratio of Rab7 relative to GAPDH. Rab7 protein increased at $24 \mathrm{~h}$ compared with sham groups, significantly, and attained a peak on day 3 , and decreased at 2 days. (b) Semiquantitative analysis of the intensity of staining of Rab7 to GAPDH. The data are means $\pm \operatorname{SEM}(n=3, \star \star P<0.01$, significantly different from the sham group). 

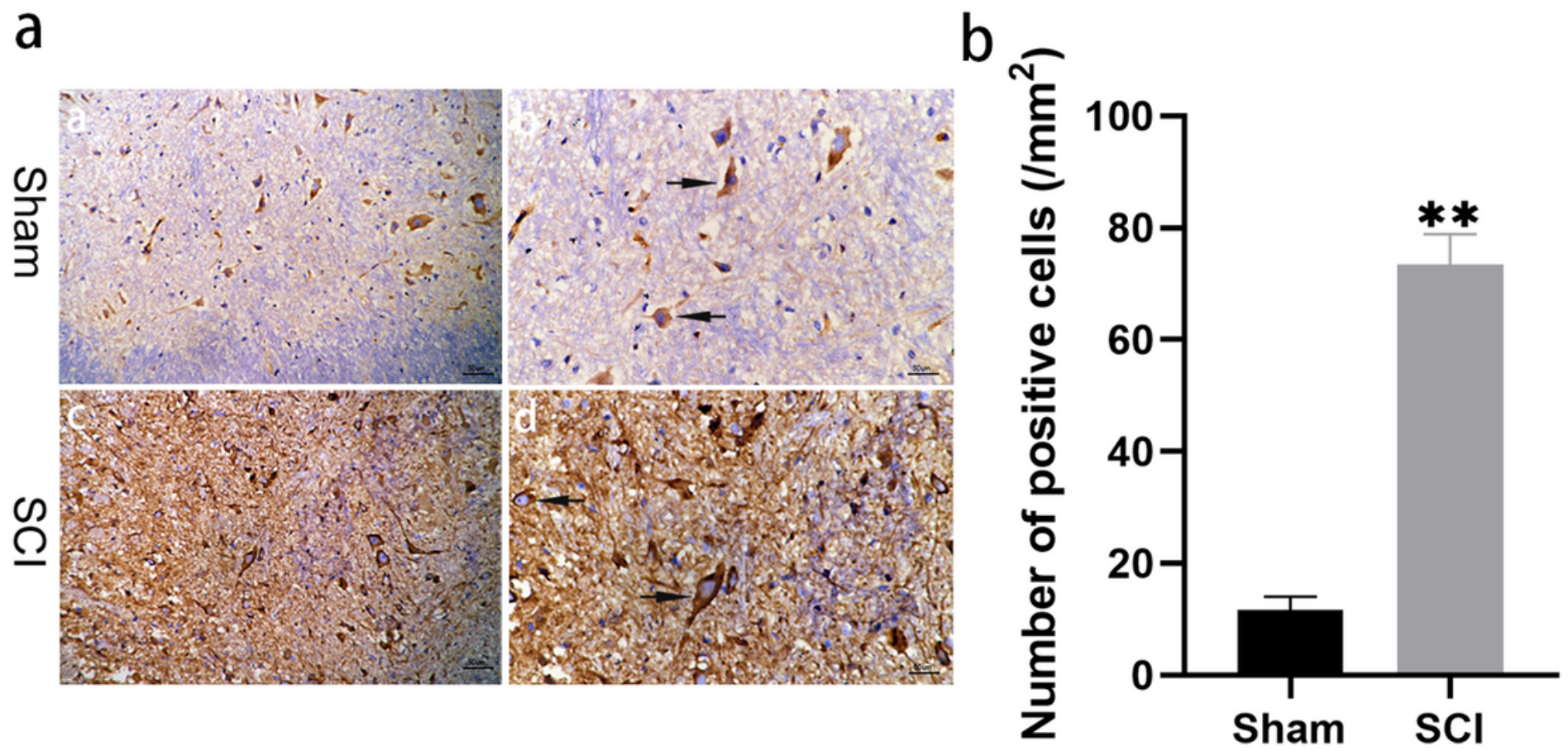

Figure 2

Immunohistochemistry expression of Rab7 in the spinal cord surrounding the wound after SCl.

Higher-power views of sections immunostained with antibody for Rab7 in the spinal cord of sham group (b) and (d) injury group. Low-power views in the spinal cord of sham group (a) and injury group (c). Quantification of Rab7-positive cells between sham and injury spinal cord in 3 days. Rab7 was significantly increased in the spinal cord at $3 \mathrm{~d}$ after trauma(e). ${ }^{*} \mathrm{P} \otimes 0.05$, differences were significant compared with the sham group at day 3 after trauma. Arrows denote Rab7 positive cells. Scale bars 50 $\mu \mathrm{m}$. 

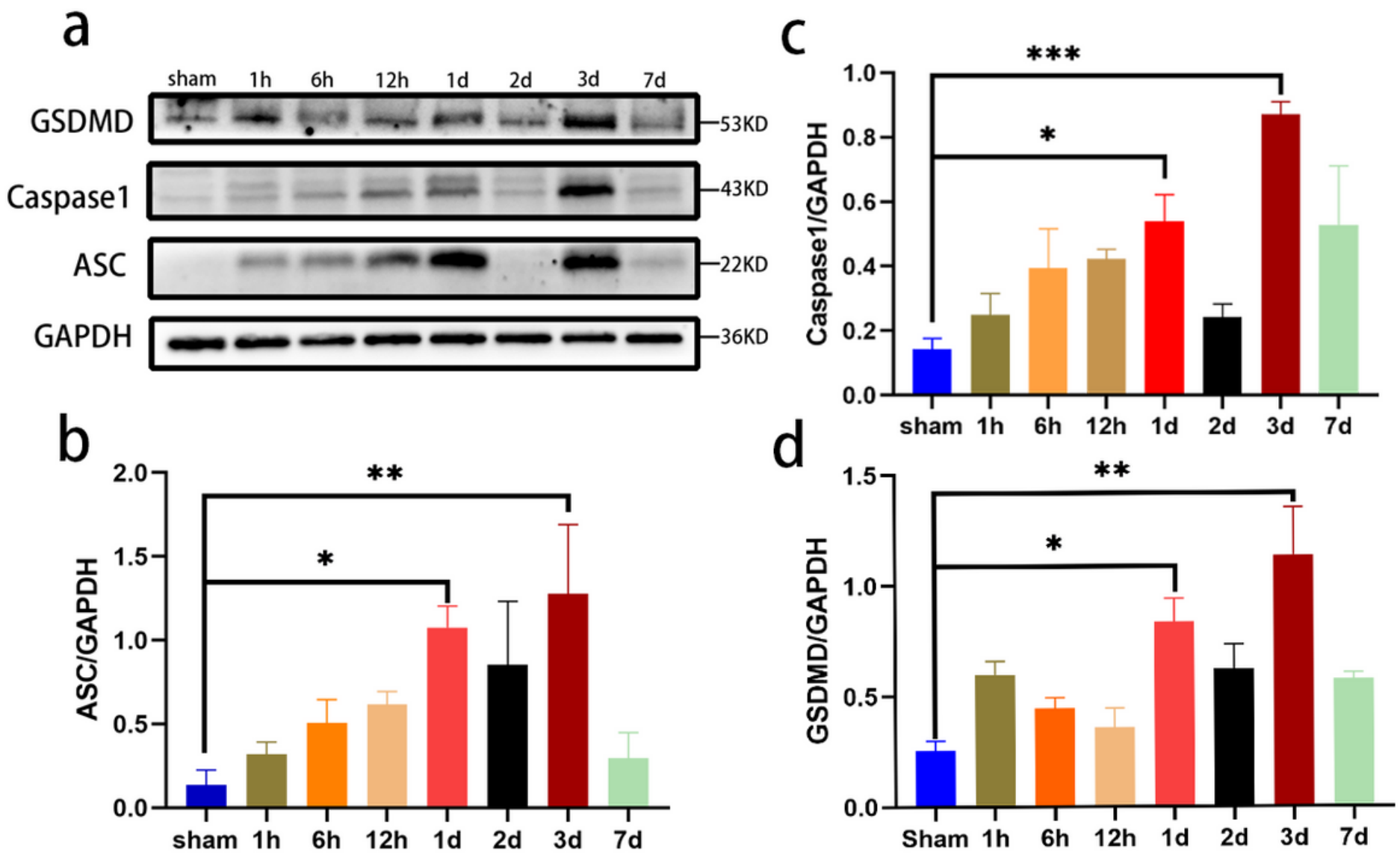

Figure 3

Western blot analysis of pyroptosis-related proteins (ASC, Caspase-1, GSDMD) in the spinal cord surrounding the wound after trauma.

Sample immunoblots probed for pyroptosis-related proteins (ASC, Caspase-1, GSDMD) is shown above. The proteins expression was increased at $24 \mathrm{~h}$ after injury and it attained a peak level at day 3 after SCl and decreased at 2 days. Quantification graphs (relative optical density) of the intensity of staining of pyroptosis-related proteins (Caspase-1, ASC, GSDMD) to GAPDH. GAPDH was compared to confirm an

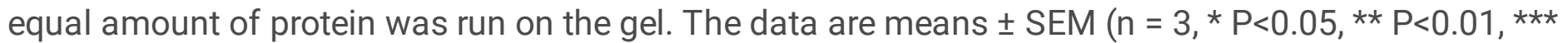
$\mathrm{P}<0.01$, significantly different from the sham group). 


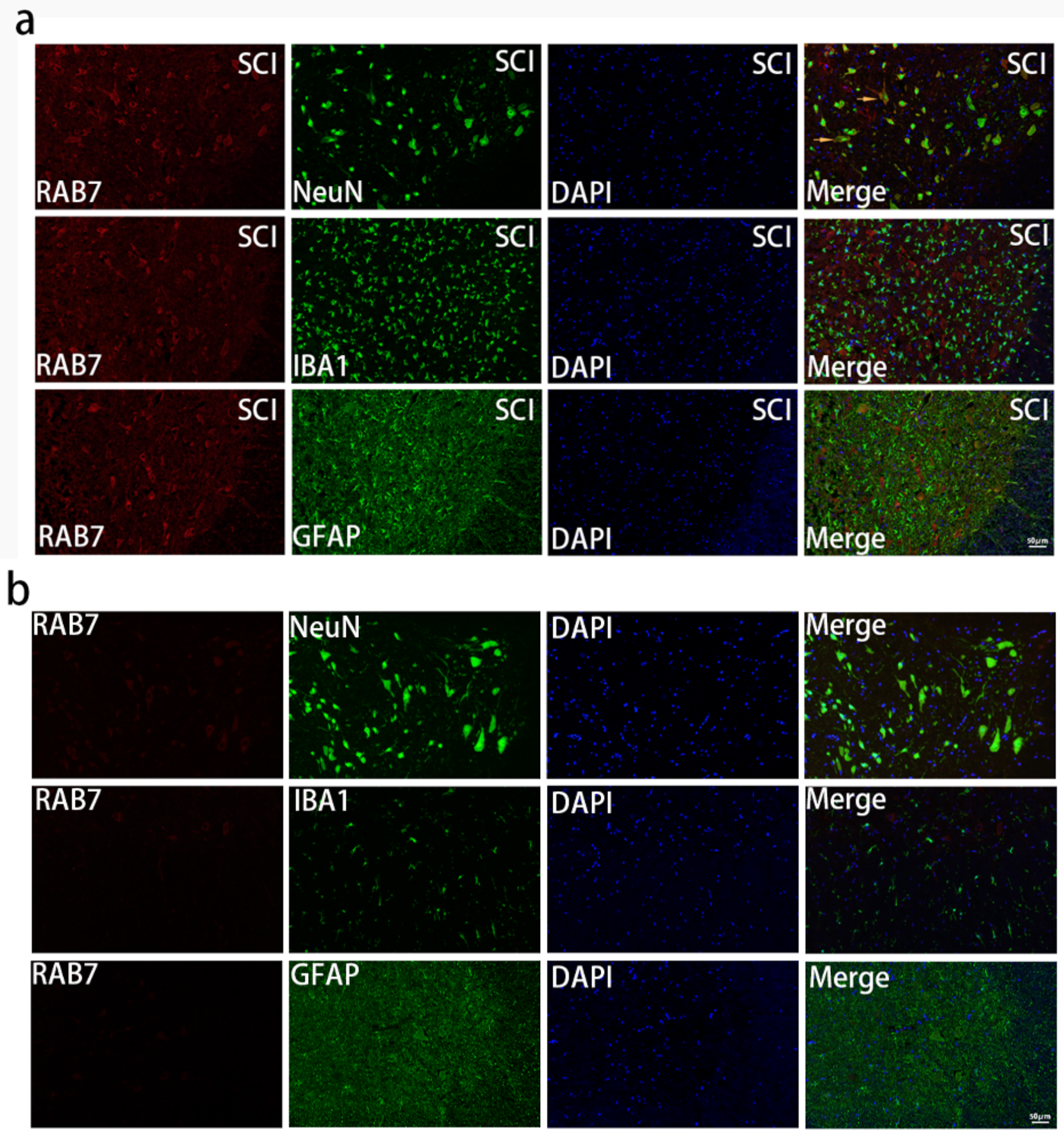

Figure 4

Double immunofluorescence staining for Rab7 and cell marker (NeuN, GFAP, IBA1) in the spinal cord at day 3 after $\mathrm{SCl}$

The spinal cord of rats within $5 \mathrm{~mm}$ distance around the injury site on the third day after $\mathrm{SCl}$, sections labeled with Rab7 (red) and cell marker (NeuN, IBA1, GFAP) (green). Dapi was used to counter-stained 
with nuclei (blue). In the merged images, the yellow color represented the colocalization of Rab7 with NeuN. Double immunofluorescent staining showed that Rab7, compared with the sham group, was significantly increased in the spinal cord at 3d after injury, and Rab7 and IBA1 or GFAP were not colocalized. Scale bars $50 \mu \mathrm{m}$.

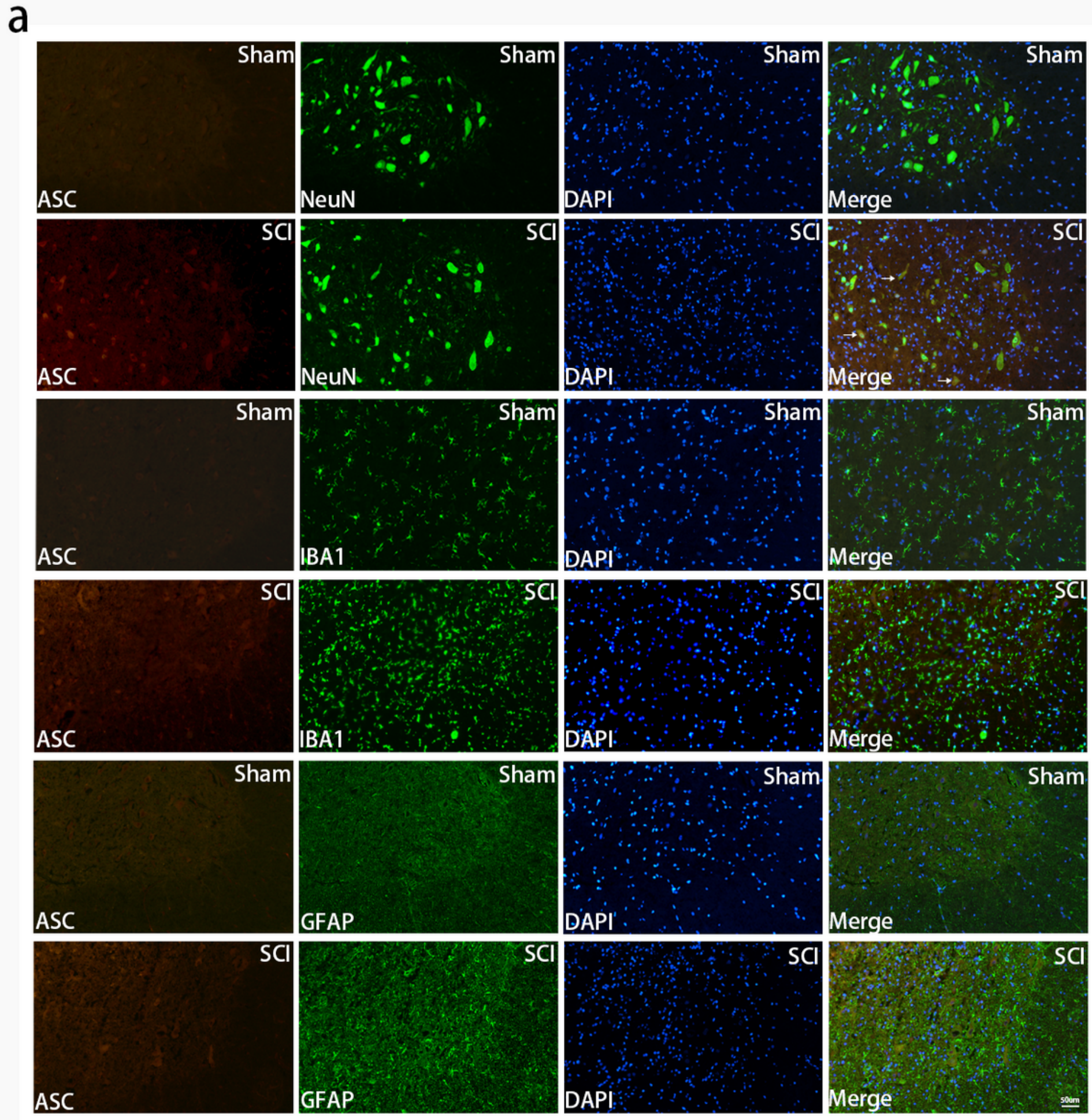

Figure 5 
Double immunofluorescence staining for ASC and cell marker (IBA1, GFAP, NeuN) in the spinal cord Sections were labeled with cell markers (NeuN, GFAP, IBA1) (green) and ASC (red). Dapi was used to counter-stained with nuclei (blue). In the merged images, the yellow color represented the colocalization of ASC with NeuN. Double immunofluorescent staining showed that ASC, compared with the sham group, was significantly increased at $3 \mathrm{~d}$ after injury. ASC and GFAP or IBA1 were not colocalized. Scale bars $50 \mu \mathrm{m}$.

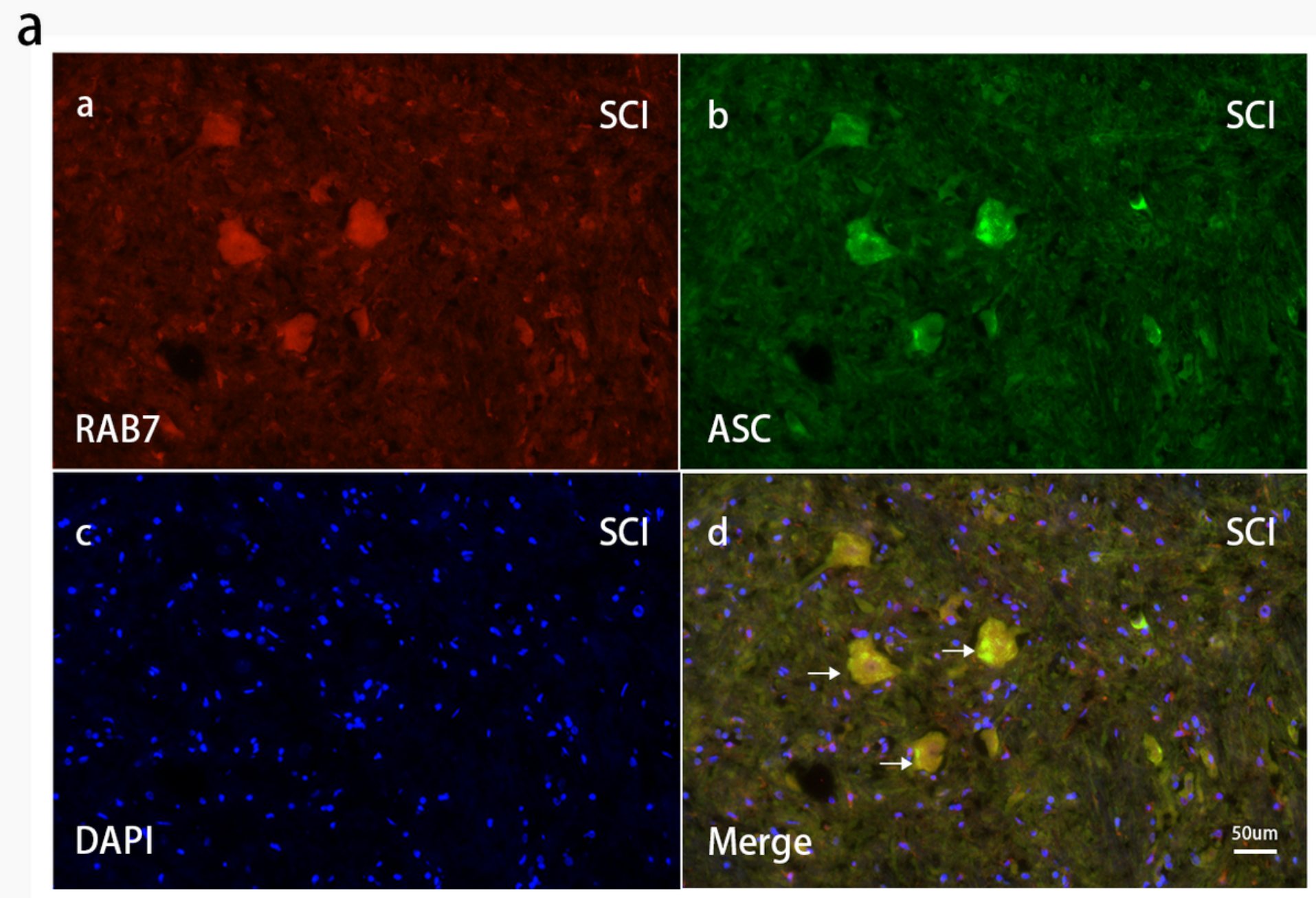

\section{Figure 6}

Double immunofluorescence staining for ASC and Rab7 in the spinal cord

The rats' spinal cord within $5 \mathrm{~mm}$ distance around the injury site on day 3 after $\mathrm{SCl}$, sections were labeled with ASC (green) and Rab7 (red). Dapi was used to counter-stained with nuclei (blue). In the merged images, the yellow color visualized represented the colocalization of ASC with Rab7. Double immunofluorescent staining demonstrated that ASC was colocalized with Rab7. Scale bars $50 \mu \mathrm{m}$. 